\title{
Ambulatory Assessment of Rumination and Worry: Capturing Perseverative Cognitions in Children's Daily Life
}

Andrea C. Kramer ${ }^{1,2}$, Andreas B. Neubauer ${ }^{1,2}$, Anja Leonhardt ${ }^{3}$, Annette Brose ${ }^{4}$, Judith $\operatorname{Dirk}^{1,2}$, and Florian Schmiedek ${ }^{1,2}$

${ }^{1}$ DIPF | Leibniz Institute for Research and Information in Education

${ }^{2}$ Center for Individual Development and Adaptive Education of Children at Risk (IDeA)

${ }^{3}$ Ludwigshafen, Germany

${ }^{4}$ Humboldt Universität Berlin

\section{Author Note}

Andrea C. Kramer (iD https://orcid.org/0000-0002-0420-5498

Andreas B. Neubauer (iD https:/ /orcid.org/0000-0003-0515-1126

Annette Brose iD https://orcid.org/0000-0001-6774-2189

Judith Dirk iD https://orcid.org/0000-0001-7224-650X

Florian Schmiedek iD https://orcid.org/0000-0001-7298-5468

This work was partly preregistered on the Open Science Framework (OSF):

https://osf.io/da5q3.

This article is partly based on data published in Dirk \& Schmiedek (2016), Dirk \& Schmiedek (2017), Galeano Weber et al. (2018), Könen et al. (2015), Könen et al. (2016), Kühnhausen et al. (2013), Neubauer et al. (2018), and Schmidt et al. (2019). It is also in part based on the dissertation completed by Anja Leonhardt (2016). We have no known conflicts of interest to disclose. 
Study 1 was part of the FLUX project at the IDeA-Center in Frankfurt a. M., Germany, which is funded by the Hessian Initiative for the Development of Scientific and Economic Excellence (LOEWE). Study 2 was funded by the Jacobs Foundation (grant number 2016-1245-00). We would like to thank Heiko Rölke and the Technology-Based Assessment Group at the DIPF for the development of the software used for the ambulatory assessment. We owe special thanks to Verena Diel, Tanja Könen, Jan Kühnhausen, Philipp Wiesemann and a team of highly committed student assistants for their help in conducting Study 1. For Study 2, we would like to thank Aamir Sajjad for further developing the software. We also owe special thanks to Michaela Menstell and a team of highly committed student assistants.

Correspondence concerning this article should be addressed to Andrea C. Kramer, DIPF | Leibniz Institute for Research and Information in Education, Rostocker Str. 6., 60323 Frankfurt am Main, Germany, Email: kramer.andrea@dipf.de

(C) 2021, American Psychological Association. This paper is not the copy of record and may not exactly replicate the final, authoritative version of the article. Please do not copy or cite without authors' permission. The final article will be available, upon publication, via its DOI: $10.1037 /$ pas0001020 


\begin{abstract}
Rumination and worry are common forms of perseverative cognitions in children. Research has started to target perseverative cognitions in the everyday life of children, however, valid measurement instruments reliably capturing rumination and worry in children's daily life are still missing. We conducted two ambulatory assessment studies validating short scales suitable for the measurement of rumination and worry in children's daily life. Results of the first study ( $N=110,8-11$-year olds, 31 days, up to 4 daily measurements) supported a unidimensional structure of the rumination scale. Rumination was associated with negative affect (but not positive affect) on the within- and on the between-person level. On the between-person level, children who ruminated more showed poorer working memory performance. In the second study $(N=84,8-10$-year olds, 21 days, up to 3 daily measurements), findings of Study 1 were largely replicated. Moreover, we established a unidimensional worry scale in Study 2 reliably capturing worry in children's daily life. Importantly, Study 2 showed that worry and rumination share common variance but can be differentiated in children. On the within-person level, higher levels of worry were associated with higher levels of negative affect and lower levels of positive affect. On the betweenperson level, worry was associated with higher levels of negative affect and lower working memory performance. Altogether, findings of both studies demonstrated that the short scales had excellent psychometric properties suggesting that they are helpful tools for the assessment of rumination and worry in children's daily life.
\end{abstract}

Keywords: rumination, worry, affective well-being, scale development, ambulatory assessment 
The present work evaluated two short scales measuring rumination and worry in children's daily life. Results supported the validity and reliability of the two short scales and showed that fluctuations of rumination and worry are related but distinct phenomena in children. These measurement instruments provide valuable tools for research on children's experiences in everyday life. They might also be useful in clinical studies that track intervention-related changes in worry and rumination. 


\section{Ambulatory Assessment of Rumination and Worry: Capturing Perseverative Cognitions in Children's Daily Life}

Perseverative cognitions are repeatedly occurring thoughts activating a mental representation of a stressor (Brosschot et al., 2006). These thoughts can refer to past stressful events as well as future anticipated stressors. Thus, the term perseverative cognitions sometimes also referred to as unconstructive repetitive thoughts (e.g., Watkins, 2008) or repetitive negative thoughts (e.g., Ehring \& Watkins, 2008) - subsumes several phenomena such as worry, rumination, or intrusive thoughts (Brosschot et al., 2006). There are various measures for different types of perseverative cognitions (e.g., worry or rumination) and most of these measures were designed to capture stable between-person differences. Yet, perseverative cognitions fluctuate dynamically in individuals' daily life (e.g., Kircanski et al., 2015), necessitating the development of reliable and valid measurement instruments of within-person fluctuations in perseverative cognitions. Although previous research has begun to target perseverative cognitions in children, valid and reliable instruments for measuring its different aspects in children's everyday life are still scarce. In the current work, we address this gap and present two short scales suitable to measure within-person rumination and worry in children's daily life.

\section{Similarities and Differences of Rumination and Worry}

Rumination is usually defined as recurring thoughts about depressive symptoms, their causes, and their consequences (Nolen-Hoeksema, 1991). Rumination is thought to generate, maintain, and prolong depressed affect (Nolen-Hoeksema \& Morrow, 1993). Several reviews and meta-analyses have reported an association between rumination and depression (e.g., Aldao et al., 2010; Mor \& Winquist, 2002; Olatunji et al., 2013). According to leading definitions, worry refers to negative, recurring, and to some extent uncontrollable thoughts about events that may happen in the future and that may have possible negative consequences 
(Borkovec et al., 1983). Worry represents a core feature of General Anxiety Disorder (American Psychiatric Association, 2013).

Both, rumination and worry, are characterized by negative and repetitive thoughts. Further, worry and rumination are abstract forms of thinking characterized by overgeneralization (Goldwin \& Behar, 2012; Stöber, 1998; Watkins et al., 2000) and are related to cognitive inflexibility and attentional bias to negative stimuli (Davis \& NolenHoeksema, 2000; Donaldson et al., 2007; Goodwin et al., 2017; Molina et al., 1998). Given the similarity of the two constructs, empirical studies revealed positive correlations between rumination and worry (Segerstrom et al., 2000; van Rijsoort et al., 1999; Watkins, 2004) and that both predict the onset and maintenance of anxious and depressed mood (see Watkins, 2008). Studies in children have linked rumination as well as worry to symptoms of anxiety and depression (Broeren et al., 2011; but see Muris et al., 2004; Verstraeten et al., 2011).

Despite these similarities, worry and rumination can be separated on the conceptual level. One of the major differences between the two constructs is the temporal orientation with rumination being mainly focused on the past, whereas worry relates to the future (e.g., McLaughlin et al., 2007; Watkins et al., 2005). In addition, research has shown that rumination revolves around self-worth, personal loss, or failure whereas worries are concerned with the anticipation of future threats that may or may not occur (Nolen-Hoeksema et al., 2008). Another difference concerns the functions of worry and rumination: Rumination is often used in the attempt to better understand one's feelings and problems, thereby trying to find a solution to or withdraw from the problematic situation at hand (Lyubomirsky \& Nolen-Hoeksema, 1993; Nolen-Hoeksema et al., 2008; Papageorgiou \& Wells, 2001). In contrast, worry is supposed to help prepare for future threat (Borkovec et al., 1999). Next to these conceptual differences, there is some evidence that rumination and worry can also be separated psychometrically (Fresco et al., 2002; but see Segerstrom et al., 2000). 
Taken together, there is considerable overlap between worry and rumination, yet these phenomena can be distinguished on the grounds of temporal orientation, content, and function. Research in children and adolescents has corroborated these findings but is still inconclusive. Worry and rumination have been shown to be highly correlated in children (Broeren et al., 2011; Verstraeten et al., 2011). Yet, in 12 - 17-year olds, rumination and worry items were shown to load on different factors (Muris et al., 2004).

\section{Assessment of Rumination and Worry}

Past research has predominantly focused on the assessment of rumination or worry as traits, that is, the general tendencies to ruminate or worry. A prominent measure of rumination is the Ruminative Responses Scale (RRS; Treynor et al., 2003), a self-report measure assessing the extent to which a person responds with reflection or brooding to depressed or negative mood. For children, there are several measures available such as the Ruminative Responses Subscale of the Children's Response Style Questionnaire (CRSQ-Rumination; Abela et al., 2000) or the Rumination Subscale of the Children's Response Style Scale (CRSS; Ziegert \& Kistner, 2002), both of which are based on the RRS. These instruments have previously been applied in children as young as six and seven years, respectively (Flancbaum et al., 2011; Harmon et al., 2019). Regarding worry, one of the most commonly used measures of between-person differences is the Penn State Worry Questionnaire (PSWQ; Meyer et al., 1990). The PSWQ captures the intensity and uncontrollability of worries and has also been adapted for children and adolescents from six to 18 years (PSWQ-C; Chorpita et al., 1997).

Different types of measurement instruments are needed, however, to capture state rumination and worry, and to measure within-person variation in these constructs (i.e., days or moments with more or less intense rumination or worry than usual). Different state measures in laboratory studies or in everyday life (i.e., in ambulatory assessment studies) 
have been proposed, and these typically comprise measures with only one or two items (e.g., Hilt \& Pollak, 2012; Kircanski et al., 2015). In ambulatory assessment studies with adults, perseverative cognitions are typically assessed as either momentary experiences (e.g., Kircanski et al., 2015), or as retrospective assessments across a certain time frame (e.g., the last day; Zoccola et al., 2011; or during the past hour; Newman et al., 2019). Ambulatory assessment studies with children captured rumination or worry using retrospective assessments referring to the past hour (Stone et al., 2019) or to the past day (Jellesma et al., 2009). Similar to studies with adults, only few items were used here to measure perseverative cognitions.

A major caveat of the current state measures of rumination and worry that are used in ambulatory assessment studies is, however, that these are not validated as are measurement instruments for trait variation (but see Brose et al., 2020). Particularly for research in children, the measurement instruments mentioned above consist of a maximum of two items and psychometric work on their properties is often missing. Consequently, more elaborate measures of within-person variation in rumination or worry are still needed. They need to fulfil three criteria: (1) capture the constructs more broadly, providing a more nuanced picture than two-item measures possibly can; (2) be short to avoid overly burdening participants, and (3) have good psychometric properties at the within-person level (Brose et al., 2020). Hence, researchers need to find a trade-off between using few items and still capturing the full breadth of a given construct. In addition, when it comes to research in children, items need to be easy to understand. Thus, the goal of the present paper is to validate two short scales in German language suitable for the comprehensive assessment of rumination and worry in children's everyday life. We do so by examining (1) the factor structure and internal consistency of two proposed measures in a multilevel structural equation modeling framework and (2) the association of these measures with two outcomes that have been 
reported to be associated with worry and rumination in prior research: affective well-being and working memory performance.

\section{Associations of Rumination and Worry with Affect}

Rumination and worry should be related to negative affect by definition as both phenomena can be described as thoughts about negative content. Research has consistently shown positive associations between worry or rumination and indicators of negative mood. These were found in (a) cross-sectional studies with a focus on more stable individual differences in rumination, worry, and/or negative affectivity (i.e., the constructs' trait components; Borkovec et al., 1983; Hofmann et al., 2005; Marchetti et al., 2018; Verstraeten et al., 2011), (b), experimental studies manipulating worry and rumination and examining effects on affective states (i.e., momentarily experienced feelings; Marchetti et al., 2018; McLaughlin et al., 2007), and (c) ambulatory assessment studies with a specific focus on within-person associations between rumination or worry and negative affect (Moberly \& Watkins, 2008; Newman et al., 2019). Findings in children are largely comparable with rumination and/or worry being linked to higher levels of negative affect (Jellesma et al., 2009; Verstraeten et al., 2011).

Regarding positive affect, findings on the association with rumination and worry have been less consistent: trait rumination (Marchetti et al., 2018) but not trait worry (Hofmann et al., 2005) was negatively associated with trait positive affect. Regarding state positive affect, previous studies have shown negative relations to rumination and worry (McLaughlin et al., 2007), however, other studies have reported no meaningful associations (e.g., Llera \& Newman, 2010; Marchetti et al., 2018). In children, research on the relation of positive affect and rumination or worry is limited. In a cross-sectional study with primary school children, worry but not rumination was negatively correlated with positive affectivity, a temperamental trait (Verstraeten et al., 2011). 


\section{Associations of Rumination and Worry With Working Memory}

Rumination and worry have both been linked to impairments in executive functions (e.g., Moran, 2016; Yang et al., 2017; Zetsche et al., 2018), a set of cognitive processes implicated in the regulation of thoughts and actions (Miyake et al., 2000). They comprise several cognitive functions, with task-set shifting, inhibition, and working memory updating representing the most commonly examined abilities. In the present work, we focus on working memory updating, which is usually conceptualized as the ability to hold and simultaneously manipulate several pieces of information in a controlled and coordinated way (Baddeley \& Hitch, 1994).

On a theoretical level, the resource allocation model (Ellis \& Ashbrook, 1988) proposes a negative relationship of rumination or worry with working memory performance. The theory posits that the negative recurrent thoughts, such as rumination and worry, consume cognitive resources. As cognitive resources are limited, processes relevant to the actual task at hand are therefore compromised. In relation to working memory, prior research has yielded some support for this prediction for both rumination (e.g., Bernblum \& Mor, 2010; Meiran et al., 2011; but see Yang et al., 2017) and worry (e.g., Gustavson \& Miyake, 2016; Moran, 2016).

In children, to our knowledge only one study examined these relations and found higher levels of trait worry to be associated with more problems in working memory and other executive functions in 7 - 12-year old children (Geronimi et al., 2016). Of note, Geronimi et al. (2016) used parents' self-reports to measure problems in children's executive functions. With regard to rumination, we are not aware of any study examining associations with working memory in children. However, in adolescents trait rumination was not significantly related to working memory performance, neither in cross-sectional nor in prospective studies (Connolly et al., 2014; Wagner et al., 2015). 


\section{The Present Work}

The aim of the present work was to develop and validate two short scales measuring rumination and worry, respectively, in children's daily life, providing valuable new tools for ambulatory assessment studies. The short scales should be easy to understand for children, capture fluctuations within children in rumination and worry, and exhibit good psychometric properties. Additionally, the short scales should capture both constructs broadly, allowing for a time-efficient but nevertheless nuanced assessment of rumination and worry in children's everyday life. To that end, we conducted two ambulatory assessment studies. In Study 1, we developed and validated the rumination short scale and tested the association of rumination with affective well-being as well as working memory performance at the within- and between-person level of analysis. As such, we examined the following hypotheses:

H1: Items of the rumination short scale represent one common underlying construct; that is, the items load on a single factor on the within- and on the between-person level, respectively.

$\mathrm{H} 2$ : More daily rumination is associated with higher negative affect and lower positive affect on the within-person level. The strength of these associations differs between children.

H3: More daily rumination is associated with lower levels of working memory performance on the within-person level. The strength of this effect varies between children.

With Study 2, we attempted to replicate the findings of Study 1, to validate the short scale assessing momentary worry as well as to test whether worry and rumination can be differentiated in children. Therefore, we examined the following hypotheses in Study 2:

H4: Findings on the structure of the rumination short scale as well as on associations with affect and working memory performance (H1-H3) are replicated. 
H5: Items of the worry short scale capture one common construct; that is, all items load on a single factor on the within- and on the between-person level, respectively.

H6: Worry and rumination are positively correlated. However, worry and rumination represent distinct constructs and thus, their latent factor correlation is smaller than 1.

H7: More momentary worries are related to higher levels of negative affect and lower levels of positive affect on the within-person level. Children differ in the strength of these effects.

H8: More momentary worries are related to lower levels of concurrent working memory performance. The strength of this within-person association varies between children.

H5-H8 were preregistered on the Open Science Framework (https://osf.io/da5q3). H2, $\mathrm{H} 3, \mathrm{H} 7, \mathrm{H} 8$, and partly $\mathrm{H} 4$ referred to the within-person level only. Therefore, analyses on the between-person level need to be considered exploratory.

\section{Study 1}

\section{Methods}

\section{Participants}

A sample of 110 children (45 girls) was recruited from an elementary school in an urban area in Germany. Children were 8-11 years old $(M=9.88, S D=0.60)$ and $77 \%$ of them were German native speakers.

\section{Procedure}

Data was collected for four weeks (i.e., up to 31 consecutive days) during the school year in May 2012 via ambulatory assessment, which means that children answered items during their daily routines. Parents gave written informed consent. The study was approved by the ethics committee of the local university.

Children received study smartphones, which did not provide access to regular functions of a smartphone (i.e., calls, text messages, WiFi, camera, etc.). They completed up 
to four assessments per day on these smartphones scheduled at 8.50 a.m., at 11:25 a.m., at 3:00 p.m., and at 7:00 p.m. Whereas school sessions were scheduled to fixed times for all children, timing of afternoon and evening sessions was adapted individually (i.e., within a time window of $+/-2$ hours). The start of these daily assessment sessions was indicated by vibration of the smartphone and a signal tone and children were instructed to answer the presented items within $60 \mathrm{~min}$ of the signal. Each of the four daily sessions lasted for about 10 to 15 min. Regarding the measures relevant for the present work, $73.6 \%$ of all first daily sessions, $73.9 \%$ of all second daily assessments, $64.5 \%$ of all third daily sessions, and $63.7 \%$ of all last daily assessments were completed. The proportion of missing values was not related to age, gender, or mean level of rumination. Extensive pre- and posttests were conducted at school in the children's classrooms before and after the daily sessions, including instruction, assessments of demographic data, trait measures, and working memory baseline measurements. See Dirk and Schmiedek (2016) for additional details. Children received money or a gift card in compensation for their study participation with the amount depending on the session time and the individual compliance (with a maximum compensation of $78.75 €)$.

\section{Measures}

Rumination. We developed the ambulatory rumination scale (ARS) to assess rumination in children's daily life. To that end, we adapted four items from the Rumination Subscale of the CRSS (Ziegert \& Kistner, 2002). In addition, two items were adapted from the Rumination Subscale of the German Coping Questionnaire for children and adolescents (SVF-KJ; Hampel et al., 2001). To capture daily rumination, items were modified by including the word today as a reference. As such, the six rumination items assessed thoughts about one's feelings and related unwanted thoughts. Children answered these items once per day in the evening. Agreement with each item was measured on a 5-point-scale from "not at 
all true" (1) to "completely true" (5). For item wording and descriptive statistics, please see Table S1 in the Online Supplemental Material.

Affect. We assessed negative and positive affect using three items each (i.e., afraid, miserable, unhappy for negative affect and good, fantastic, content for positive affect). These items have been tested in prior work, which demonstrated their appropriateness in ambulatory assessment studies with children (see Leonhardt et al., 2016). Children were asked to indicate how they felt at the moment on a 5-point scale ranging from "not at all true" (1) to "completely true" (5). To compute negative and positive affect scores, we averaged the respective items at each occasion. Reliability was estimated as McDonald's $\omega$ (Geldhof et al., 2014) for the within- and the between-person level. Reliability for negative affect (positive affect), was estimated as .75 (.71) on the within-person level and $.99(.85)$ on the betweenperson level.

Working Memory. Children completed a numerical and a spatial updating task three times per day (in the morning, shortly before noon, and in the afternoon). A detailed description of the tasks and information on psychometric properties in the present sample have been reported in Dirk and Schmiedek (2016). Both tasks were presented on the smartphone and each task consisted of four trials with a memory load of two (Load 2 condition) and four trials with a memory load of three (Load 3 condition).

In the numerical updating task, children had to memorize two (or three) digits (in the range from 0 to 9) and perform additions and subtractions within the range of +2 and -2 on each of the digits. In the spatial updating task, children had to keep track of the position of two (or three) differently colored cartoon creatures in a 4 x 4 grid. In the Load 2 conditions, three updating operations were presented whereas in the Load 3 conditions, children had to perform four updating operations. For each measurement occasion and task, we computed the mean accuracy of the eight responses in the Load 2 condition ( $4 \times 2$ responses) and of the 12 
responses in the Load 3 condition ( 4 x 3 responses). To ensure continuous task processing, mean accuracy scores were computed only if children had given at least one response per trial in each load condition. Mean accuracy scores for each load condition and each task were then averaged into a total working memory accuracy score per measurement occasion. Reliability ${ }^{1}$ (McDonald's $\omega$; Geldhof et al., 2014) was .68 on the within-person level and .94 on the between-person level.

\section{Data Analysis}

In the present study, we treated repeated measurements (Level 1) as nested within children (Level 2) resulting in a hierarchical structure of the data which was accommodated by applying multilevel models (Raudenbush \& Bryk, 2002) for analyses.

H1: Structure of the ARS. To evaluate whether the ARS reliably captures withinand between-person variation of rumination in children, we conducted a two-level confirmatory factor analysis (CFA; Chou et al., 2000). This approach allows for estimating the within-person and between-person models simultaneously by separating the total variance into within-person and between-person variance components. To test if our six items fit a common-factor model of rumination, we conducted a two-level CFA, with all items loading on one common factor. To evaluate the model fit, we used different fit indices with conventional cut-off criteria for acceptable model fit: the root mean square error of approximation (RMSEA; cut-off: $\leq .08)$, the comparative fit index (CFI; cut-off: $\geq .90)$, and the standardized root mean square residual (SRMR; cut-off: $\leq .08$ ). The latter index can be estimated separately for the within- and the between-part of the model, respectively. We deemed model fit acceptable if at least three out of the four model fit indicators (CFI, RMSEA, SRMR within, $_{\text {SRMR }}$ between) suggested acceptable model fit.

\footnotetext{
${ }^{1}$ Reliability was estimated using the model reported in Dirk and Schmiedek (2016). In this model residuals of the spatial updating task (Load 2 and Load 3) were correlated on both levels of analysis. In addition, the variance of the numerical task (Load 3) was fixed to 0 on the between-person level.
} 
H2: Associations of Rumination with Same-Day Negative and Positive Affect. We estimated 2 two-level models to evaluate the effect of daily rumination on daily negative and positive affect. Daily negative or positive affect was computed as the average of the four affect ratings across the day. Child $j$ 's affect at day $d\left(\right.$ affect $\left._{d j}\right)$ was then predicted by this child's daily rumination ( rumination $_{d j}$ ), centered on child $j$ 's person-mean of rumination to obtain a pure within-person effect (e.g., Raudenbush \& Bryk, 2002). Child j’s affect was also predicted by this child's mean of all rumination reports (rumination. pmean $_{j}$ ), centered on the grand-mean. Random effects were included in the model for the intercept as well as for the effect of rumination and were allowed to co-vary freely (unstructured G-matrix).

Likelihood ratio tests were used to evaluate significance of random effects, comparing the fit of the model with vs. without the respective random variance. The model can be described using the following equations:

Level 1 (within children):

$$
\operatorname{affect}_{d j}=\beta_{0 j}+\beta_{1 j} \cdot \text { rumination }_{d j}+\varepsilon_{d j}
$$

Level 2 (across children):

$$
\begin{gathered}
\beta_{0 j}=\gamma_{00}+\gamma_{01} \cdot \text { rumination. pmean }{ }_{j}+v_{0 j} \\
\beta_{1 j}=\gamma_{10}+v_{1 j}
\end{gathered}
$$

We ran two models, one with daily negative affect and one with daily positive affect as dependent variable.

\section{H3: Associations of Rumination with Same-Day Working Memory Performance.}

To test the effects of rumination on working memory performance, we estimated the same model as described in Equations 1-3. However, as the dependent variable we used daily working memory performance $\left(\mathrm{wmp}_{d j}\right)$, which was computed as the average of each child's working memory accuracy scores per day. In addition to the person-mean centered rumination variable (rumination ${ }_{d j}$ ) and the grand-mean centered person-mean of rumination 
(rumination. pmean $_{j}$ ), we included a variable indicating study day to control for effects of time (e.g., practice effects). This variable was centered on each child's first day of the study. Multilevel structural equation modeling was performed in Mplus (version 8.3; Muthén \& Muthén, 1998-2017). All other analyses were conducted in R version 3.5.1 for Windows (R Core Team, 2018). Multilevel models were estimated using the nlme package (Pinheiro et al., 2018). For all analyses, we used the conventional $\alpha$ level of .05 (two-tailed).

\section{Results and Discussion Study 1}

Descriptive statistics of variables in Study 1 can be found in Table 1. On the withinperson level, rumination was correlated with negative affect $(r=.18, p<.001)$, negative affect was negatively correlated with positive affect $(r=-.17, p=.001)$ as well as with working memory performance $(r=-.18, p<.001)$ and positive affect was positively correlated with working memory performance $(r=.13, p=.002)$. On the between-person level, the same pattern of correlations was found (see Table 1).

\section{H1: Structure of the ARS}

The multilevel CFA of the one-dimensional rumination factor model resulted in the following fit indices: $\mathrm{CFI}=.89, \mathrm{RMSEA}=.07, \mathrm{SRMR}_{\text {within/between }}=.05 / .06$. Figure $1 \mathrm{a}$ depicts the schematic representation of the measurement model of the ARS. As described in the methods section, we deemed model fit satisfactory because three (RMSEA, SRMR within, $\mathrm{SRMR}_{\text {between }}$ ) out of four fit indices suggested acceptable model fit ${ }^{2}$. Factor loadings were high at the between-person level and moderate to high at the within-person level (see Figure 1a). All factor loadings were statistically significant indicating systematic common variance across items and justifying the computation of a rumination score across all items. On both levels, reliability was good or excellent in the present sample $\left(\omega_{\text {within }}=.84\right.$, $\left.\omega_{\text {between }}=.96\right)$.

\footnotetext{
${ }^{2}$ Model fit could be further improved by including the covariance of Items r04 and $\mathrm{r} 05$ as well as of Items r05 and r06 on the within- and between-person level, $\chi^{2}(4)=133.56, p<.001$. This model had excellent model fit, $\mathrm{CFI}=.98, \mathrm{RMSEA}=.03, \mathrm{SRMR}_{\text {within/between }}=.02 / .03$.
} 
Taken together, these findings suggest that the items of the ARS represent one common underlying construct and reliably assess rumination on the between-person as well as on the within-person level.

\section{H2: Associations of Rumination with Same-Day Negative and Same-Day Positive Affect}

In multilevel models, daily negative and daily positive affect, respectively, were predicted from daily rumination and person average rumination (see Equations 1 to 3 ). Model results are depicted in Table 2 . In the model with daily negative affect as the dependent variable, the effect of rumination was statistically significant on the within- and on the between-person level, $b=.085, p<.001$, and $b=.295, p<.001$, respectively. This indicates that on days when children reported higher levels of daily rumination than typical for them, they also reported higher negative affect. Further, children reporting higher levels of rumination compared to the average of the sample also reported higher levels of negative affect. The random effect for daily rumination was also statistically significant, $\chi^{2}(2)=72.97$, $p<.001$, indicating that the link between daily rumination and daily negative affect varied in strength between children. These findings dovetail with previous findings on the betweenperson level in child samples using trait-like conceptualizations of negative affect (i.e., children who report higher levels of rumination also report higher levels of negative affectivity; Verstraeten et al., 2011). In addition, our results extend these findings to the within-person level showing that the positive association of rumination and negative affect also holds within children.

In the model using daily positive affect as the dependent variable, the effects of rumination were not statistically significant. Contrary to our hypothesis, children did not report lower levels of positive affect on days when they reported higher levels of rumination than usual, $b=-0.007, p=.785$. As such, these findings may suggest that rumination has differential effects on the two dimensions of affective well-being targeted in the present work 
(i.e., negative and positive affect). A likelihood ratio test comparing a model with vs. without the random effect of daily rumination showed that the random effect of rumination was statistically significant, $\chi^{2}(2)=13.73, p=.001$. This indicates that children differed in the strength of the association between daily rumination and daily positive affect.

To test whether results change when controlling for the effects of time, we conducted sensitivity analyses including a variable indicating the study day in both models. This did not change pattern of results in both models (see Online Supplemental Material, Table S2).

\section{H3: Associations of Rumination with Same-Day Working Memory Performance}

Next, we set up a two-level model predicting daily working memory performance from daily rumination and person average rumination as well as time (i.e., study day). In contrast to our hypothesis, this model (see Table 2) revealed that on days when children reported higher levels of rumination than usual their working memory performance was not lower than usual, $b=0.007, p=.326$. However, the between-person effect of rumination was statistically significant, $b=-.055, p=.043$, indicating that children who generally reported higher levels of rumination also showed poorer working memory performance (compared to the average child in the sample). The random effect of daily rumination was statistically significant, $\chi^{2}(2)=36.32, p<.001$, implying that children differed in the strength of the link between daily rumination and daily working memory performance.

Results on the between-person level are generally in line with between-person findings in adult populations (e.g., Bernblum \& Mor, 2010; Meiran et al., 2011). Regarding the lack of a meaningful average within-person effect of daily rumination on daily working memory performance, a potential explanation may be the design of the study: Daily rumination was assessed retrospectively in the evening. As such, we do not know when exactly children engaged in rumination and therefore it is possible that children may not have 
ruminated at times when working memory performance was measured. In Study 2, we therefore measured perseverative cognitions (worry) and working memory simultaneously.

\section{Study 2}

The aims of Study 2 were (1) to replicate the findings on Study 1, and (2) to develop a measure to capture momentary worries in children.

\section{Method}

\section{Participants}

Children were recruited in Frankfurt am Main, Germany, and surrounding areas via presentation of the research project to parents or students at elementary schools, distribution of leaflets, and advertisements to parents on social media platforms. Enrollment in the study took place in two waves: The first wave started in May 2019 yielding a sample of 23 children (of whom two children decided not to continue with the study during the first day of ambulatory assessment). In accordance with the preregistration (see https://osf.io/da5q3), we conducted a second wave of enrollment in October 2019 to increase the sample size. Sixtythree children participated in the second wave of enrollment yielding a total sample of $N=84$ (37 girls) children ${ }^{3}$. Children were $8-10$ years old $(M=8.98$ years, $S D=0.78)$. Most children $(84 \%)$ started to learn German within the first two years of life.

\section{Procedure}

Study 2 consisted of a 21-day ambulatory assessment design embedded in an introductory session and a posttest, which were conducted at our laboratory. The study was approved by a local ethics committee.

\footnotetext{
${ }^{3}$ As specified in our preregistration, we attempted to recruit 100 children. However, despite increased effort to attain this number, we could not recruit more than 84 participants in the two waves of data collection that we had specified. Following our preregistration, we performed an additional power analysis using the same population parameters as in our initial power analysis (50\% compliance, a small within-person effect of momentary worries accounting for $1 \%$ of the within-person variance in negative affect in combination with a random effect indicating that more than $80 \%$ of participants would have a positive within-person association between worry and negative affect). Estimated power with the updated number of participants was $97.6 \%$.
} 
Parents provided written informed consent for their own and their children's participation. Children provided written informed assent. While parents filled in a background questionnaire, children received their study smartphone and completed the introductory session, which consisted of detailed explanations of the study procedure, how to operate the study smartphones, and how to respond to the items as well as how to solve the working memory tasks. Additionally, children answered demographic as well as trait questionnaires and completed working memory baseline measurements. The introductory session was conducted in small groups of up to six children and took approximately $90 \mathrm{~min}$. Children kept their study smartphone for the entire ambulatory assessment phase. As in Study 1, smartphones were specially programmed so that only our study application providing the questionnaires and working memory tasks was available.

For each child, the ambulatory assessment phase of the study lasted 21 days starting the day after the introductory session had been completed. During the ambulatory assessment phase, children completed up to three assessments per day, one in the morning, one in the afternoon, and one in the evening. To accommodate children's individual daily routines, assessments were scheduled within specific time frames, in which they could be completed. Additionally, parents could choose between two options determining the timing of the afternoon and the evening session. During the week, the morning session was available between 6:45 am and 7:50 am (completion time approximately $3 \mathrm{~min}$ ). At weekends, children could complete the first daily session until 9:15 am. The afternoon (evening) session was available either between 3:00 pm and 5:15 pm $(6: 00 \mathrm{pm}$ and 8:30 pm) or between 4:00 pm and 6:15 pm (7:00 pm and 9:30 pm), depending on parents' choices. During the afternoon session, children completed self-report measures as well as working memory tasks. Completion of the afternoon session took approximately $10 \mathrm{~min}$. In the evening session, children provided self-reports, which took approximately $7 \mathrm{~min}$. Smartphones vibrated or 
rang in the afternoon and in the evening to indicate the start of the respective session. In the morning, smartphones remained silent as not to disturb the children's individual sleep routines.

Within five weekdays after the end of the ambulatory assessment phase, children reported to our laboratory again, returned the smartphones, and filled in a final set of questionnaires consisting of self-report measures and feedback questions lasting approximately $20 \mathrm{~min}$. As compensation for their participation in the study, parents received $5 €$ and children received a gift card in the amount of $25 €$. If children had completed more than $70 \%(90 \%)$ of all assessments, they received an additional gift card in the amount of $5 €$ (10€). Of the potential 1,764 morning sessions (84 participants x 21 days), children responded to the measures relevant for the present work at 1,506 sessions, corresponding to a compliance rate of $85.4 \%$. Similarly, $79.7 \%$ of the afternoon sessions and $89.6 \%$ of the evening sessions were filled-in. The proportion of missing values was not related to age, gender, or mean level of rumination or worry, respectively.

\section{Measures}

In the following, we provide information on measurements relevant for the present analyses. Information on the full set of variables assessed in the study can be found online (https://osf.io/v829s/)

Worry. We developed a set of seven items to assess momentary levels of worries in children, the ambulatory worry scale (AWS). Development of these items was based on established trait measures of worry in children such as the PSWQ-C (Chorpita et al., 1997; German version: PSWQ-KF; Adam \& Hoyer, 2003) as well as single items used in previous ambulatory assessment studies with adults (Kircanski et al., 2015; Scott et al., 2015). Items were also developed based on the different aspects of worry as described in the definition (e.g., Borkovec et al., 1983). Thus, next to occurrence and intensity of worry the items 
covered the aversive nature of thoughts, the repetitiveness and uncontrollability, as well as the future orientation. Developed items were short and easy to understand for children. A pilot study ( $N=55$, age range: 7-10 years) testing children's comprehension of items did not reveal any problems. Responses were given on a 5-point Likert-Scale ranging from "not at all true" (1) to "completely true" (5). Current levels of worries were assessed in the afternoon as well as in the evening session. For item wording and descriptive statistics, please see Table S3 in the Online Supplemental Material.

Affect. Children reported on their momentary affective state three times per day (in the morning, afternoon, and evening). Negative affect was assessed using the items "Right now, I feel unhappy (miserable/ sad/ afraid/ nervous/ uneasy).”. Items used to measure positive affect were "Right now I feel good (fantastic/ content/ active/ interested/ enthusiastic).”. Responses were given on a 5-point Likert-Scale ranging from "not at all true" (1) to "completely true" (5). Negative and positive affect scores were computed as the average of the respective items at each occasion. Reliability for negative affect (positive affect) was .78 (.79) on the within-person level and $.95(.87)$ on the between-person level.

Rumination. As in Study 1, children filled in the ARS each evening. Daily levels of rumination were computed using the average of the six items.

Working Memory Performance. In the afternoon session, children completed the same working memory tasks as in Study 1 with identical load conditions and identical numbers of trials per task and occasion. Details on the configuration of the tasks (i.e., presentation times, interstimulus intervals, etc.) can be found online (https://osf.io/da5q3). Mean working memory accuracy scores were computed as in Study 1. Reliability on the within-person level was $\omega=.53$, and $\omega=.86$ on the between-person level.

\section{Data Analysis}


Similar to Study 1, we used two-level models to accommodate the nested hierarchical structure of the data. All analyses reported for $\mathrm{H} 5-\mathrm{H} 8$ were preregistered (https://osf.io/da5q3). Deviations from the preregistration are explicitly noted.

H4: Replication of Results of Study 1. To test whether the results from Study 1 could be replicated, we performed the analyses for $\mathrm{H} 1, \mathrm{H} 2$, and $\mathrm{H} 3$ using the sample of Study 2. Daily negative and positive affect scores were computed as the average across the three measurement occasions per day. Daily working memory performance corresponded to the mean accuracy score of the respective measurement occasion (afternoon occasion) per day.

H5: Structure of the AWS. To evaluate whether the items of the worry scale represent one common construct, we conducted a multilevel CFA (a one-factor model with all seven items loading on one common factor). To evaluate model fit, we used the same fit indices and cut-off criteria as for the CFA of the ARS in Study 1.

H6: Association of Worry with Rumination. To evaluate the validity of the worry scale, we examined the associations between worry and rumination using two-level structural equation modeling. To model the latent factors worry and rumination we used the final measurement models for the AWS and the ARS. As rumination was assessed once per day (in the evening), we averaged the two measurement occasions for the worry items to obtain a daily score for each item, which, in turn, was used in the two-level structural equation models. To evaluate whether worry and rumination are related but distinct constructs, we compared two models: In the first model, the correlation between both latent factors (worry and rumination) was freely estimated. In the second model, the correlation was constrained to 1, implying that worry and rumination represent the same construct (note that this second model is equivalent to a model in which all items load on one common factor). Since the two models are nested, they can be compared using a likelihood ratio test, adjusted by a scaling correction factor when using the robust maximum likelihood estimator (Yuan \& Bentler, 
2000). A significant likelihood ratio test would indicate that the more complex model (i.e., the model with two correlated factors) provides a better fit to the data and thus, should be preferred.

\section{H7: Associations of Worry with Concurrent Negative Affect and Concurrent}

Positive Affect. To analyze the associations between momentary worry and concurrent affect, we estimated the same models as for $\mathrm{H} 2$ with momentary affect replacing daily affect and momentary levels of worry replacing daily rumination.

H8: Association of Worry with Concurrent Working-Memory Performance. To examine the effect of worry on working memory performance, we set up the same model as for H3 with daily rumination being replaced by momentary levels of worry (and with personmean rumination being replaced by person-mean worry).

\section{Results and Discussion Study 2}

Descriptive statistics are presented in Table 1. On the within-person level, negative affect was positively correlated with rumination $(r=.28, p<.001)$ and with worry $(r=.58, p$ $<.001)$. There was a positive relation between rumination and worry $(r=.36, p<.001)$ but neither rumination nor worry correlated with working memory performance $(r=.04, p$ $=.283$, for rumination, and $r=.00, p=.986$, for worry). However, there was a small positive correlation between working memory performance and positive affect $(r=.12, p=.003)$. On the between-person level, negative affect was highly correlated with rumination $(r=.66, p$ $<.001)$ and worry $(r=.77, p<.001)$ and moderately correlated with working memory performance $(r=-.38)$. Rumination and worry were also highly correlated $(r=.69, p<.001)$ and both correlated with working memory performance ( $r=-.33$, for rumination and worry).

\section{H4: Replication of Results of Study 1}


All analyses performed in Study 1 were performed again using the data of Study 2. First, we fit a multilevel CFA with all rumination items loading on one common factor ${ }^{4}$. With all fit indices suggesting acceptable model fit $\left(\mathrm{CFI}=.91, \mathrm{RMESA}=.06, \mathrm{SRMR}_{\text {within/between }}\right.$ $=.04 / .04)$, the unidimensional structure of the ARS was replicated, as was the high internal consistency, $\omega_{\text {within }}=.85, \omega_{\text {between }}=.98$. Factor loadings were similar as in Study 1 (see Online Supplemental Material, Table S4). Second, multilevel models were used to test whether daily negative affect and daily positive affect were predicted from daily rumination. The pattern of results was the same as in Study 1: on the within-person (as well as on the between-person) level, daily rumination predicted daily negative affect, $b=0.167, p<.001$ ( $b$ $=0.385, p<.001)$, but not daily positive affect, $b=-0.011, p=.782(b=0.088, p=.306)$. Likewise, children differed in the strength of the link between daily rumination and negative affect, $\chi^{2}(2)=59.16, p<.001$, and positive affect, $\chi^{2}(2)=64.59, p<.001$, respectively. Controlling for time trends did not change these patterns. Next, we evaluated whether working memory performance was predicted by daily rumination. Again, results from Study 1 were replicated in Study 2: Working memory performance was linked to rumination on the between-person level, $b=-0.054, p=.003$, but not on the within-person level, $b=0.001, p$ $=.822$. In contrast to Study 1 , the random effect of rumination was not statistically significant, $\chi^{2}(2)=1.68, p=.431$. Detailed results can be found in Table 2. Taken together, findings in Study 2 largely replicated the findings from Study 1, supporting the unidimensional structure as well as the validity and reliability of the ARS in an independent sample of children within approximately the same age range.

\section{H5: Structure of the AWS}

\footnotetext{
${ }^{4}$ Please note that we fit exactly the same model as the initial model in Study 1, that is, without error covariances between items.
} 
The multilevel CFA with one worry factor on the within- and on the between-person level revealed a good fit in the present sample, $\mathrm{CFI}=.965, \mathrm{RMSEA}=.026, \mathrm{SRMR}_{\text {within/between }}$ $=.024 / .006$. The measurement model of the AWS is schematically depicted in Figure 1b. Significant factor loadings of all items implied systematic common variance across items and justified averaging the items into one common worry score. Following our preregistered decision guidelines, we deemed the model fit good. Reliability on the within- and the between person level was excellent in the present sample $\left(\omega_{\text {within }}=.93\right.$, $\left.\omega_{\text {between }}=.98\right)$.

\section{H6: Association of Worry with Rumination}

The comparison ${ }^{5}$ of the two models yielded an implausible negative $\chi^{2}$-difference value, most likely due to estimation problems. However, comparing the fit indices (CFI,

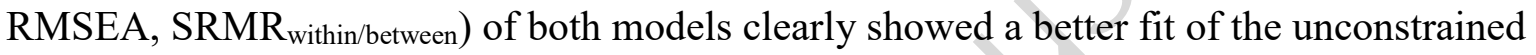
model $^{6}$. In order to obtain an interpretable model comparison, we refitted both models using the maximum likelihood estimator (instead of the robust maximum likelihood estimator). This step had not been preregistered. Model comparison indicated a statistically significant better fit of the unrestricted model (i.e., the factor correlation was freely estimated), $\chi^{2}(2)=$ $3123.03, p<.001$, indicating that worry and rumination are related but distinct constructs: on the within-person level, the correlation between the latent factors worry and rumination was $.38, p<.001$, and on the between-person level, it was .73, $p<.001$. Additional exploratory analyses further showed that restricting the correlation on the between-person level to 1 only revealed similar results: the unrestricted model had a statistically significant better fit, $\chi^{2}(1)=280.05, p<.001$, indicating that worry and rumination are separable on both levels.

\footnotetext{
${ }^{5}$ We used the MplusAutomation package (Hallquist \& Wiley, (2018) for model comparisons.

${ }^{6}$ Fit indices of the unconstrained model were as follows: $\mathrm{CFI}=.94, \mathrm{RMSEA}=.04, \mathrm{SRMR}_{\text {within/between }}$ $=.04 / .05, \mathrm{BIC}=39084.85, \mathrm{AIC}=38723.09$. For the constrained model, fit indices were as follows: $\mathrm{CFI}=.56, \mathrm{RMSEA}=.10, \mathrm{SRMR}_{\text {within/between }}=.17 / .14, \mathrm{BIC}=42193.08, \mathrm{AIC}=41842.12$.
} 


\section{H7: Associations of Worry with Concurrent Negative Affect and Concurrent Positive Affect}

Next, we set up two-level models predicting positive affect and negative affect, respectively, from momentary worries and person average worries (see Table 3). In line with our hypothesis, there was a statistically significant within-person effect of worry on negative affect, $b=.388, p<.001$. This means that when children reported higher levels of worry than typical for them, they also reported higher levels of negative affect. In addition, the effect of worry on the between-person level was also statistically significant, $b=.673, p<.001$, meaning that children generally reporting higher levels of worry (than the average child in the sample) also reported higher levels of negative affect. Further, there were between-person differences in the within-person effect of worry on negative affect, $\chi^{2}(2)=493.09, p<.001$.

For positive affect, only the within-person, $b=-.128, p=.044$, but not the betweenperson effect of worry, $b=.035, p=.785$, was statistically significant. This indicates that when children reported higher levels of worry than usual, they also reported lower levels of positive affect. However, children generally reporting higher levels of worry did not show higher or lower levels of positive affect. There was significant random variance in the withinperson effect of worry, $\chi^{2}(2)=224.65, p<.001$.

As preregistered, we conducted sensitivity analyses controlling for time trends. This did not change the pattern of results (see Online Supplemental Material, Table S5).

\section{H8: Association of Worry with Concurrent Working Memory Performance}

Contrary to our hypothesis, the within-person effect of worry was not statistically significant, $b=-.003, p=.678$ (see Table 3 ). A likelihood ratio test comparing this model to a model without the random variance of worries revealed that the random effect was not statistically significant, $\chi^{2}(2)=0.58, p=.748$. On the between-person level, the effect of worry was statistically significant, $b=-.089, p=.003$. Thus, children reporting higher levels 
of worry than the average child in the sample also showed poorer working memory performance.

\section{Exploratory Analyses}

Differentiation Between Rumination and Worry. By testing a model with rumination and worry items loading on a common factor against a model with rumination and worry items loading on two distinct but correlated factors (on both levels), we investigated if rumination and worry can be differentiated in children. However, another approach to differentiate these two constructs would be to examine a bi-factor model with all items loading on a general factor as well as all rumination items loading on a rumination-specific factor and all worry items loading on a worry-specific factor (with all factors being uncorrelated). Results of this exploratory analysis can be found in the Online Supplement (Tables S6-S7). Comparing the fit indices of the bi-factor model to the correlated two-factor model indicated that the bi-factor model had a better fit than the two-factor model. A likelihood ratio test of the two nested models (see Yung et al., 1999) revealed a better fit of the bi-factor model than the two-factor model, $\chi^{2}(24)=340.280, p<.001$. In the bi-factor model, all worry items displayed statistically significant loadings on the general as well as on the worry-specific factor on both levels, with loadings on the specific factor being numerically higher. Regarding the rumination items, all loadings were statistically significant for the general factor on both levels, but not for the rumination-specific factor. Specifically, on the within-person level item r05 did not display a significant loading on the ruminationspecific factor and on the between-person level loadings of items r05 and r06 were not statistically significant. Given the content of these items (i.e., thoughts that one cannot get rid of), the general factor in the bi-factor model, representing the common ground between rumination and worry, may reflect repetitiveness of thoughts. Regarding the remaining factors, the rumination-specific factor seems to represent rumination about feelings and 
negative events. The worry-specific factor seems to reflect aspects of uncontrollability, aversiveness, and possible negative outcomes. Hence, both factors seem to reflect the defining criteria of the rumination and worry constructs. Taken together, the bi-factor model provides further evidence for common features of rumination and worry as well as distinct aspects differentiating the two constructs.

\section{Independent Associations of Rumination and Worry With Different Aspects of}

Negative Affect. To provide further evidence that rumination and worry can be distinguished in children, we tested whether both constructs would independently predict negative affect. Additionally, we tested whether both predictors would be differentially related to different aspects of negative affect, that is, high- or low-arousal negative affect as well as the different aspects of negative affect represented by the six single items. To that end, we estimated nine multilevel models containing rumination as well as daily worry (i.e., the mean across the two measurement occasions per day) as predictors on the within- as well as on the betweenperson level. The dependent variable was either daily negative affect, daily high-arousal negative affect (i.e., the daily mean of the items "nervous", "afraid", "uneasy"), daily lowarousal negative affect (i.e., the daily mean of the items "unhappy", "miserable", "sad") or the daily mean of each single negative affect item. On both levels of analysis, rumination and worry independently predicted daily negative affect, daily high- and low-arousal negative affect as well as four out of the six single items of daily negative affect (unhappy, nervous, miserable, and sad). However, rumination was not significantly related to the item "afraid" on the within-person level as well as to the item "uneasy" on both levels. Consequently, these items may be more closely related to worry than rumination. Detailed results can be found in the Online Supplement (Table S8).

Shortening the ARS and the AWS. In the context of ambulatory assessment, measurement instruments usually consist of very few items, and single items measures are 
not uncommon in this context. As we have argued before, such measures may not provide a nuanced picture of the construct of interest, however, time-efficient measurement is a key element in ambulatory assessment. To that end, we examined if the number of items of the ARS and the AWS can be reduced without compromising too much on capturing both constructs in their entirety. As a result, item r02 of the ARS and items w03 and w04 of the AWS were excluded as the content of these items showed some overlap to other items of the respective scale. We then repeated the analyses reported for Study 2 using the 5-item versions of the ARS and the AWS. Pattern of results remained largely the same. The only exception was the within-person effect of worry on positive affect, which was not significant when using the 5-item version of the AWS, $b=-.102, p=.097$. Detailed results on these additional exploratory analyses can be found in the Online Supplemental Material (Tables S9-S13 and S14). In sum, these results show that the shorter versions of the ARS and the AWS are also appropriate tools to capture rumination and worry in children's daily life.

\section{General Discussion}

In research with children to date, short measurement instruments comprehensively capturing within-person fluctuations in rumination and worry are scarce. Therefore, the main aim of the present work was to develop and validate two such measures capturing withinperson variation of rumination and worry in children's daily life. We tested these instruments in two ambulatory assessment studies in children aged $8-11$ years.

\section{Psychometric Properties of the ARS and the AWS}

The ARS and the AWS exhibited good psychometric properties: When examined individually, both measurement instruments showed a unidimensional factor structure. In addition, internal consistency was high for both scales and on both levels indicating that the ARS as well as the AWS reliably assess differences between children in rumination or worry and also reliably capture within-person variation of rumination and worry within children. 
Study 2 further revealed that rumination and worry are psychometrically separable in children: The latent correlation between worry and rumination was moderate on the withinperson level and large, but significantly smaller than 1 on the between-person level. Shared variance of the two latent factors amounted to $14.4 \%$ on the within-person level and to $53.3 \%$ on the between-person level. This finding illustrates that worry and rumination overlap to some extent but nevertheless represent distinct constructs in children. This conclusion was further supported by exploratory analyses indicating that a bi-factor model with a general factor representing repetitiveness of thoughts and a rumination- as well as a worry-specific factor fit the data well. These results integrate findings showing that worry and rumination share common variance (Segerstrom et al., 2000) and simultaneously exhibit unique characteristics (Fresco et al., 2002; Muris et al., 2004).

Taken together, the present findings suggest that it is useful to account for the common variance between both constructs when examining effects of both, rumination and worry, and to this end, a bi-factor model is a suitable modeling option. However, when the key focus is on either of the two constructs, the use of single scales and thus a unidimensional conceptualization of each construct seems appropriate. This is also important from a practical point of view as the use of both scales might not always be feasible in ambulatory assessment studies.

\section{Associations of Rumination and Worry with Negative Affect}

Results regarding the association between rumination or worry and negative affect were highly consistent across studies: Both, rumination and worry were associated with negative affect on the within- as well as on the between-person level. On days (moments) when children reported higher levels of rumination (worry), they also reported higher daily (concurrent) levels of negative affect. These findings are in line with previous research on the relation between rumination or worry and state-like negative affect (Marchetti et al., 2018; 
McLaughlin et al., 2007; Moberly \& Watkins, 2008; Newman et al., 2019). Further, children differed in the strength of this association. These differences between children suggest that some children may be particularly vulnerable to the negative effects of perseverative cognitions. This might also be relevant for psychosocial development: future studies should examine whether stronger couplings may be predictive of negative long-term developmental trajectories, such as the development of clinical symptoms or psychological disorders (e.g., Vanderhasselt et al., 2016).

In addition, children generally reporting higher levels of rumination (worry) also reported higher levels of negative affect. These findings correspond to previous findings on the relation between rumination or worry and trait-like negative affect in children as well as in adults (e.g., Borkovec et al., 1983; Marchetti et al., 2018; Verstraeten et al., 2011). Additionally, these findings reflect the definitions of rumination and worry characterizing both constructs as thoughts about negative content (e.g., Borkovec et al., 1983; NolenHoeksema, 1991). Thus, we interpret these positive associations between rumination or worry and negative affect on the within- as well as on the between-person level as evidence for the validity of both short scales.

Besides, exploratory analyses revealed the predictive distinctness between rumination and worry, showing that the two constructs independently predicted daily negative affect as well as its different subtypes or facets (i.e., high- and low-arousal negative affect or single items). Exceptions were the items "afraid" and "uneasy", which seem to be more consistently linked to worry than rumination. This is in line with worry representing a core feature of anxiety (American Psychiatric Association, 2013) as well as research showing that worry is associated with prolonged physiological activity (e.g., Brosschot et al., 2007; Pieper et al., 2010), which may be more closely related to subjective feelings of uneasiness. 
On the between-person level, associations of perseverative cognitions with positive affect have also been highly consistent across both studies. Rumination was not associated with positive affect in either study, meaning that children who reported higher levels of rumination in general did not report lower levels of positive affect. Similarly, children who reported higher levels of worry in Study 2 in general did not report lower levels of positive affect. These findings on the between-person level are partly consistent with prior research: A previous study also reported no association between rumination and positive affect in children but found evidence for an association between worry and positive affect (Verstraeten et al., 2011). However, it is important to acknowledge the different conceptualizations of positive affect: Whereas Verstraeten et al. (2011) measured positive affectivity as a temperamental trait, we conceptualized positive affect on the between-person level as an aggregate across all momentary ratings of positive affect within the study period.

Our results thus seem to indicate that the two different dimensions of affective wellbeing, positive and negative affect, differentially relate to perseverative cognitions. Theories such as the Perseverative Cognition Hypothesis (Brosschot et al., 2006) or the Contrast Avoidance Model (Newman \& Llera, 2011) suggest that perseverative cognitions prolong negative emotions, for example, to avoid a sudden increase in negative affect. As such, individuals who ruminate or worry a lot may maintain a high level of negative emotions. At the same time, these individuals do not seem to have difficulties with processing positive emotions (Llera \& Newman, 2010). We note, however, that our preregistered hypotheses specifically referred to the within-person associations of rumination / worry with affective well-being. Hence, the findings on the between-person level need to be interpreted cautiously and should be replicated in future research.

On the within-person level, daily rumination was, contrary to our hypothesis, not linked to daily levels of positive affect. However, in line with our hypothesis, the strength of 
this effect differed between children indicating that for some children higher levels of daily rumination were associated with lower levels of daily positive affect. This pattern of results was exactly replicated in Study 2. In accordance with expectations, worry was negatively related to positive affect within children across time, and the strength of this association also varied between children. This means that at moments when children reported higher levels of momentary worries than typical for them they also reported lower concurrent positive affect. One potential explanation for the different results regarding the associations of rumination and worry with positive affect on the within-person level may result from the different time scales of the ARS and the AWS. Whereas the ARS assesses retrospective evaluations of daily rumination, the AWS measures momentary levels of worry. Retrospective evaluations may not be perfectly accurate and refer to different experienced moments during the day than the momentary assessments of affect (Neubauer et al., 2020). Thus, using concurrent assessments of momentary experiences may facilitate detecting these within-person effects on positive affect, which might be smaller than corresponding associations with negative affect.

\section{Associations of Rumination and Worry with Working Memory Performance}

Findings on the within-person association between perseverative cognitions and working memory were highly consistent across studies and across the different types of perseverative cognitions: Neither rumination nor worry were associated with working memory performance on the within-person level. Thus, neither on days when children reported ruminating more than usual nor in situations when children reported higher levels of worries than typical, working memory performance was compromised. In Study 1, children differed in the strength of the effect of rumination on working memory performance, however, in Study 2 we did not find evidence for meaningful variation between children, 
neither for the effect of rumination nor for the effect of worry on working memory performance ${ }^{7}$.

Generally, these findings are not in line with the resource allocation model (Ellis \& Ashbrook, 1988) predicting impairment of working memory performance when engaging in perseverative cognitions. Nevertheless, a few aspects merit consideration: In both studies, daily levels of rumination were assessed retrospectively at the end of the day whereas working memory performance was assessed at different times during the day. With the lack of concurrent assessment of both constructs, we cannot rule out the possibility that children may have engaged in rumination at some point during the day but not at times when working memory performance was assessed. Thus, we may not have captured the effect of momentary rumination on working memory in both studies. To specifically test the concurrent effect of perseverative cognitions on working memory performance, we assessed worry and working memory performance concurrently in Study 2. As we did not find evidence for a meaningful concurrent effect of worry on working memory performance, this finding stands in further contrast to the resource allocation model. We hasten to add that the lack of the within-person effect may also be explained in terms of reliability of working memory performance on the within-person level, which was comparatively low in Study $2(\omega=.53$, vs. $\omega=.68$ in Study 1). Thus, the lack of association on the within-person level needs to be interpreted carefully and cannot unequivocally refute predictions of the resource allocation model. Further research targeting the within-person association of perseverative cognitions and working memory using more extensive assessments is needed before strong conclusions can be drawn.

On the between-person level, results of both studies revealed the same pattern for both types of perseverative cognitions. Children reporting higher levels of rumination or worry in general showed poorer working memory performance. Overall, results were consistent across

\footnotetext{
${ }^{7}$ This may be due to the smaller sample size and consequently lower power in Study 2 .
} 
the two studies and are in line with some previous findings in child and adult populations (Bernblum \& Mor, 2010; Geronimi et al., 2016; Gustavson \& Miyake, 2016; Meiran et al., 2011; Moran, 2016) but differ from studies in adolescents, which reported no significant associations between rumination and working memory (Connolly et al., 2014; Wagner et al., 2015). We note that, as the focus of the present work was primarily the development of two short scales assessing rumination and worry in children's daily life, we did not explore the association between working memory and perseverative cognitions in further detail, which would, for example, require more exploratory analyses on potential moderators of this association and ideally confirmatory replications in independent samples. Furthermore, we specifically targeted associations on the within-person level and did not specify confirmatory hypotheses targeting between-person relations. Thus, we refrain from further interpreting these between-person associations

\section{Limitations}

The studies presented in the current work are limited in several ways: First, rumination was assessed retrospectively in both studies. In comparison, outcome variables used in the analyses were assessed at different time points throughout the day and thus, contemporary within-person associations may have been obscured by the design of the studies. This also extends to the correlation between rumination and worry as levels of worries were measured referring to the present moment. Thus, the correlation on the withinperson level between both types of perseverative cognitions could be higher if both variables were measured simultaneously and referring to the same time frame. Second, in Study 2 reliability of working memory performance was comparatively low. Therefore, results on the within-person effects of rumination or worry on working memory performance were likely attenuated. The lack of an average within-person effect for both types of perseverative cognitions may not accurately reflect processes on the within-person level. Future studies 
should therefore use a more extensive measurement of ambulatory working memory performance to reliably capture within-person variance in working memory performance.

\section{Conclusions}

Providing a reliable and valid assessment of rumination and worry in children's daily life, the ARS and the AWS, respectively, may foster further research targeting for example the within-child dynamics in perseverative cognitions. Future studies may examine temporal dynamics such as the course of worry throughout the day or levels of rumination throughout the week. Especially in clinical research the ARS and the AWS may be useful to investigate, for example, changes in rumination or worry in the course of therapy. Further, changes in worry or rumination in relation to changes in affective well-being may extend the findings presented here and advance our understanding of the dynamic interplay of these phenomena in children's daily life. Of course, there are ample interesting and important research questions relating to the realm of children's everyday rumination or worry. The ARS and the AWS constitute appropriate tools to measure these phenomena and thus may help to contribute to our growing understanding of perseverative cognitions in children.

With the present work, we present two useful tools for ambulatory assessment of rumination and worry in children. The two scales, the ARS and the AWS, reliably captured within- and between-person differences in rumination and worry. Associations with affective well-being and working memory performance were generally very consistent not only across the two different studies conducted in the present work but also across the two different types of perseverative cognitions, rumination and worry. At the same time, study findings also showed that worry and rumination overlap but have distinct features. The high consistency in findings across two independent child samples further supports the validity of the present results. Further, these findings highlight the importance to carefully separate between- from within-person effects. 


\section{References}

Abela, J. R. Z., Rochon, A., \& Vanderbilt, E. (2000). The Children's Response Styles Questionnaire (Unpublished questionnaire). Montreal, Canada: McGill University.

Adam, S., \& Hoyer, J. (2003). PSWQ: KF - Penn State Worry Questionnaire - Kinderform. PSYNDEX Tests Info.

Aldao, A., Nolen-Hoeksema, S., \& Schweizer, S. (2010). Emotion-regulation strategies across psychopathology: A meta-analytic review. Clinical Psychology Review, 30(2), $217-$ 237. https://doi.org/10.1016/j.cpr.2009.11.004

American Psychiatric Association. (2013). Diagnostic and statistical manual of mental disorders $\left(5^{\text {th }}\right.$ ed. $)$. Author.

Baddeley, A. D., \& Hitch, G. J. (1994). Developments in the concept of working memory. Neuropsychology, 8(4), 485-493. https://doi.org/10.1037/0894-4105.8.4.485

Bernblum, R., \& Mor, N. (2010). Rumination and emotion-related biases in refreshing information. Emotion, 10(3), 423-432. https://doi.org/10.1037/a0018427

Borkovec, T. D., Hazlett-Stevens, H., \& Diaz, M. L. (1999). The role of positive beliefs about worry in generalized anxiety disorder and its treatment. Clinical Psychology \& Psychotherapy, 6(2), 126-138. https://doi.org/10.1002/(SICI)10990879(199905)6:2<126::AID-CPP193>3.0.CO;2-M

Borkovec, T. D., Robinson, E., Pruzinsky, T., \& DePree, J. A. (1983). Preliminary exploration of worry: Some characteristics and processes. Behaviour Research and Therapy, 21(1), 916. https://doi.org/10.1016/0005-7967(83)90121-3

Broeren, S., Muris, P., Bouwmeester, S., Heijden, Kristiaan B. van der, \& Abee, A. (2011). The role of repetitive negative thoughts in the vulnerability for emotional problems in non- 
clinical children. Journal of Child and Family Studies, 20(2), 135-148.

https://doi.org/10.1007/s10826-010-9380-9

Brose, A., De Raedt, R., \& Vanderhasselt, M.-A. (2020). Eight items of the Ruminative Response Scale are sufficient to measure weekly within-person variation in rumination. Current Psychology. Advance online publication. https://doi.org/10.1007/s12144-020$\underline{00913-y}$

Brosschot, J. F., Gerin, W., \& Thayer, J. F. (2006). The perseverative cognition hypothesis: A review of worry, prolonged stress-related physiological activation, and health. Journal of Psychosomatic Research, 60(2), 113-124.

https://doi.org/10.1016/j.jpsychores.2005.06.074

Brosschot, J. F., van Dijk, E., \& Thayer, J. F. (2007). Daily worry is related to low heart rate variability during waking and the subsequent nocturnal sleep period. International Journal of Psychophysiology, 63(1), 39-47. https://doi.org/10.1016/j.ijpsycho.2006.07.016

Chorpita, B. F., Tracey, S. A., Brown, T. A., Collica, T. J., \& Barlow, D. H. (1997). Assessment of worry in children and adolescents: An adaptation of the Penn State Worry Questionnaire. Behaviour Research and Therapy, 35(6), 569-581. https://doi.org/10.1016/S0005-7967(96)00116-7

Chou, C.-P., Bentler, P. M., \& Pentz, M. A. (2000). A two-stage approach to multilevel structural equation models: Application to longitudinal data. In T. D. Little, K. U. Schnabel, \& J. Baumert (Eds.), Modeling longitudinal and multilevel data: Practical issues, applied approaches, and specific examples (pp. 33-49). Lawrence Erlbaum Associates Publishers.

Connolly, S. L., Wagner, C. A., Shapero, B. G., Pendergast, L. L., Abramson, L. Y., \& Alloy, L. B. (2014). Rumination prospectively predicts executive functioning impairments 
in adolescents. Journal of Behavior Therapy and Experimental Psychiatry, 45(1), 46-56. https://doi.org/10.1016/i.jbtep.2013.07.009

Davis, R. N., \& Nolen-Hoeksema, S. (2000). Cognitive inflexibility among ruminators and nonruminators. Cognitive Therapy and Research, 24(6), 699-711. https://doi.org/10.1023/A:1005591412406

Dirk, J., \& Schmiedek, F. (2016). Fluctuations in elementary school children's working memory performance in the school context. Journal of Educational Psychology, 108(5), 722-739. https://doi.org/10.1037/edu0000076

Donaldson, C., Lam, D., \& Mathews, A. (2007). Rumination and attention in major depression. Behaviour Research and Therapy, 45(11), 2664-2678. https://doi.org/10.1016/j.brat.2007.07.002

Ehring, T., \& Watkins, E. (2008). Repetitive negative thinking as a transdiagnostic process. International Journal of Cognitive Therapy, 1(3), 192-205. https://doi.org/10.1521/ijct.2008.1.3.192

Ellis, H. C., \& Ashbrook, P. W. (1988). Resource allocation model of the effects of depressed mood states on memory. In K. Fiedler \& J. P. Forgas (Eds.), Affect, cognition, and social behavior (pp. 25-43). Hogrefe.

Flancbaum, M., Oppenheimer, C. W., Abela, J. R. Z., Young, J. F., Stolow, D., \& Hankin, B. L. (2011). The effects of rumination on the timing of maternal and child negative affect. Journal of Clinical Child \& Adolescent Psychology, 40(4), 596-606. https://doi.org/10.1080/15374416.2011.581615

Fresco, D. M., Frankel, A. N., Mennin, D. S., Turk, C. L., \& Heimberg, R. G. (2002). Distinct and overlapping features of rumination and worry: The relationship of cognitive production to negative affective states. Cognitive Therapy and Research, 26(2), 179-188. https://doi.org/10.1023/A:1014517718949 
Geldhof, G. J., Preacher, K. J., \& Zyphur, M. J. (2014). Reliability estimation in a multilevel confirmatory factor analysis framework. Psychological Methods, 19(1), 72-91. https://doi.org/10.1037/a0032138

Geronimi, E. M. C., Patterson, H. L., \& Woodruff-Borden, J. (2016). Relating worry and executive functioning during childhood: The moderating role of age. Child Psychiatry and Human Development, 47(3), 430-439. https://doi.org/10.1007/s10578-015-0577-4

Goldwin, M., \& Behar, E. (2012). Concreteness of idiographic periods of worry and depressive rumination. Cognitive Therapy and Research, 36(6), 840-846. https://doi.org/10.1007/s10608-011-9428-1

Goodwin, H., Yiend, J., \& Hirsch, C. R. (2017). Generalized Anxiety Disorder, worry and attention to threat: A systematic review. Clinical Psychology Review, 54, 107-122. https://doi.org/10.1016/j.cpr.2017.03.006

Gustavson, D. E., \& Miyake, A. (2016). Trait worry is associated with difficulties in working memory updating. Cognition and Emotion, 30(7), 1289-1303.

https://doi.org/10.1080/02699931.2015.1060194

Hallquist, M. N., \& Wiley, J. F. (2018). MplusAutomation: An R package for facilitating large-scale latent variable analyses in Mplus. Structural Equation Modeling: A Multidisciplinary Journal, 25(4), 621-638. https://doi.org/10.1080/10705511.2017.1402334

Hampel, P., Petermann, F., \& Dickow, B. (2001). SVF-KJ. Stressverarbeitungsfragebogen von Janke und Erdmann angepasst für Kinder und Jugendliche. Hogrefe.

Harmon, S. L., Stephens, H. F., Repper, K. K., Driscoll, K. A., \& Kistner, J. A. (2019). Children's rumination to sadness and anger: Implications for the development of depression and aggression. Journal of Clinical Child \& Adolescent Psychology, 48(4), 622-632. https://doi.org/10.1080/15374416.2017.1359788 
Hilt, L. M., \& Pollak, S. D. (2012). Getting out of rumination: Comparison of three brief interventions in a sample of youth. Journal of Abnormal Child Psychology, 40(7), 11571165. https://doi.org/10.1007/s10802-012-9638-3

Hofmann, S. G., Moscovitch, D. A., Litz, B. T., Kim, H.-J., Davis, L. L., \& Pizzagalli, D. A. (2005). The worried mind: Autonomic and prefrontal activation during worrying. Emotion, 5(4), 464-475. https://doi.org/10.1037/1528-3542.5.4.464

Jellesma, F. C., Verkuil, B., \& Brosschot, J. F. (2009). Postponing worrisome thoughts in children: The effects of a postponement intervention on perseverative thoughts, emotions and somatic complaints. Social Science \& Medicine, 69(2), 278-284. https://doi.org/10.1016/j.socscimed.2009.04.031

Kircanski, K., Thompson, R. J., Sorenson, J. E., Sherdell, L., \& Gotlib, I. H. (2015). Rumination and worry in daily life. Clinical Psychological Science, 3(6), 926-939. https://doi.org/10.1177/2167702614566603

Leonhardt, A., Könen, T., Dirk, J., \& Schmiedek, F. (2016). How differentiated do children experience affect? An investigation of the within- and between-person structure of children's affect. Psychological Assessment, 28(5), 575-585. https://doi.org/10.1037/pas0000195

Llera, S. J., \& Newman, M. G. (2010). Effects of worry on physiological and subjective reactivity to emotional stimuli in generalized anxiety disorder and nonanxious control participants. Emotion (Washington, D.C.), 10(5), 640-650. https://doi.org/10.1037/a0019351

Lyubomirsky, S., \& Nolen-Hoeksema, S. (1993). Self-perpetuating properties of dysphoric rumination. Journal of Personality and Social Psychology, 65(2), 339-349. https://doi.org/10.1037//0022-3514.65.2.339 
Marchetti, I., Mor, N., Chiorri, C., \& Koster, E. H. W. (2018). The Brief State Rumination Inventory (BSRI): Validation and psychometric evaluation. Cognitive Therapy and Research, 42(4), 447-460. https://doi.org/10.1007/s10608-018-9901-1

McLaughlin, K. A., Borkovec, T. D., \& Sibrava, N. J. (2007). The effects of worry and rumination on affect states and cognitive activity. Behavior Therapy, 38(1), 23-38. https://doi.org/10.1016/j.beth.2006.03.003

Meiran, N., Diamond, G. M., Toder, D., \& Nemets, B. (2011). Cognitive rigidity in unipolar depression and obsessive compulsive disorder: Examination of task switching, Stroop, working memory updating and post-conflict adaptation. Psychiatry Research, 185(1-2), 149-156. https://doi.org/10.1016/j.psychres.2010.04.044

Meyer, T. J., Miller, M. L., Metzger, R. L., \& Borkovec, T. D. (1990). Development and validation of the Penn State Worry Questionnaire. Behaviour Research and Therapy, 28(6), 487-495. https://doi.org/10.1016/0005-7967(90)90135-6

Miyake, A., Friedman, N. P., Emerson, M. J., Witzki, A. H., Howerter, A., \& Wager, T. D. (2000). The unity and diversity of executive functions and their contributions to complex "Frontal Lobe" tasks: A latent variable analysis. Cognitive Psychology, 41(1), 49-100. https://doi.org/10.1006/cogp.1999.0734

Moberly, N. J., \& Watkins, E. (2008). Ruminative self-focus and negative affect: An experience sampling study. Journal of Abnormal Psychology, 117(2), 314-323. https://doi.org/10.1037/0021-843X.117.2.314

Molina, S., Borkovec, T. D., Peasley, C., \& Person, D. (1998). Content analysis of worrisome streams of consciousness in anxious and dysphoric participants. Cognitive Therapy and Research, 22(2), 109-123. https://doi.org/10.1023/A:1018772104808

Mor, N., \& Winquist, J. (2002). Self-focused attention and negative affect: A meta-analysis. Psychological Bulletin, 128(4), 638-662. https://doi.org/10.1037/0033-2909.128.4.638 
Moran, T. P. (2016). Anxiety and working memory capacity: A meta-analysis and narrative review. Psychological Bulletin, 142(8), 831-864. https://doi.org/10.1037/bul0000051

Muris, P., Roelofs, J., Meesters, C., \& Boomsma, P. (2004). Rumination and worry in nonclinical adolescents. Cognitive Therapy and Research, 28(4), 539-554. https://doi.org/10.1023/B:COTR.0000045563.66060.3e

Muthén, L. K., \& Muthén, B. O. (1998-2017). Mplus user‘s guide: Eighth Edition.

Neubauer, A. B., Scott, S. B., Sliwinski, M. J., \& Smyth, J. M. (2020). How was your day? Convergence of aggregated momentary and retrospective end-of-day affect ratings across the adult life span. Journal of Personality and Social Psychology. Advance online publication. https://doi.org/10.1037/pspp0000248

Newman, M. G., Jacobson, N. C., Zainal, N. H., Shin, K. E., Szkodny, L. E., \& Sliwinski, M. J. (2019). The effects of worry in daily life: An ecological momentary assessment study supporting the tenets of the Contrast Avoidance Model. Clinical Psychological Science, 7(4), 794-810. https://doi.org/10.1177/2167702619827019

Newman, M. G., \& Llera, S. J. (2011). A novel theory of experiential avoidance in generalized anxiety disorder: A review and synthesis of research supporting a contrast avoidance model of worry. Clinical Psychology Review, 31(3), 371-382. https://doi.org/10.1016/j.cpr.2011.01.008

Nolen-Hoeksema, S. (1991). Responses to depression and their effects on the duration of depressive episodes. Journal of Abnormal Psychology, 100(4), 569-582. https://doi.org/10.1037//0021-843X.100.4.569

Nolen-Hoeksema, S., \& Morrow, J. (1993). Effects of rumination and distraction on naturally occurring depressed mood. Cognition and Emotion, 7(6), 561-570. https://doi.org/10.1080/02699939308409206 
Nolen-Hoeksema, S., Wisco, B. E., \& Lyubomirsky, S. (2008). Rethinking rumination. Perspectives on Psychological Science, 3(5), 400-424. https://doi.org/10.1111/j.1745$\underline{6924.2008 .00088 . x}$

Olatunji, B. O., Naragon-Gainey, K., \& Wolitzky-Taylor, K. B. (2013). Specificity of rumination in anxiety and depression: A multimodal meta-analysis. Clinical Psychology: Science and Practice, 20(3), 225-257. https://doi.org/10.1111/cpsp.12037

Papageorgiou, C., \& Wells, A. (2001). Metacognitive beliefs about rumination in recurrent major depression. Cognitive and Behavioral Practice, 8(2), 160-164. https://doi.org/10.1016/S1077-7229(01)80021-3

Pieper, S., Brosschot, J. F., van der Leeden, R., \& Thayer, J. F. (2010). Prolonged cardiac effects of momentary assessed stressful events and worry episodes. Psychosomatic Medicine, 72(6), 570-577. https://doi.org/10.1097/PSY.0b013e3181dbc0e9

Pinheiro, J., Bates, D., DebRoy, S., Sarkar, D., \& R Core Team. (2018). nlme: Linear and nonlinear mixed effects models (Version R package version 3.1-137) [Computer software]. https://CRAN.R-project.org/package $=$ nlme

R Core Team. (2018). R: A language and environment for statistical computing [Computer software]. R Foundation for Statistical Computing. Vienna, Austria. https://www.Rproject.org/

Raudenbush, S. W., \& Bryk, A. S. (2002). Hierarchical linear models: Applications and data analysis methods (2. ed.). Advanced quantitative techniques in the social sciences series: Vol. 1. Sage Publ.

Scott, S. B., Graham-Engeland, J. E., Engeland, C. G., Smyth, J. M., Almeida, D. M., Katz, M. J., Lipton, R. B., Mogle, J. A., Munoz, E., Ram, N., \& Sliwinski, M. J. (2015). The effects of stress on cognitive aging, physiology and emotion (ESCAPE) project. $B M C$ Psychiatry, 15, 146. https://doi.org/10.1186/s12888-015-0497-7 
Segerstrom, S. C., Tsao, J. C. I., Alden, L. E., \& Craske, M. G. (2000). Worry and rumination: Repetitive thought as a concomitant and predictor of negative mood. Cognitive Therapy and Research, 24(6), 671-688. https://doi.org/10.1023/A:1005587311498

Stöber, J. (1998). Worry, problem elaboration and suppression of imagery: The role of concreteness. Behaviour Research and Therapy, 36(7-8), 751-756. https://doi.org/10.1016/S0005-7967(98)00027-8

Stone, L. B., Mennies, R. J., Waller, J. M., Ladouceur, C. D., Forbes, E. E., Ryan, N. D., Dahl, R. E., \& Silk, J. S. (2019). Help me feel better! Ecological momentary assessment of anxious youths' emotion regulation with Parents and Peers. Journal of Abnormal Child Psychology, 47(2), 313-324. https://doi.org/10.1007/s10802-018-0454-2

Treynor, W., Gonzalez, R., \& Nolen-Hoeksema, S. (2003). Rumination reconsidered: A psychometric analysis. Cognitive Therapy and Research, 27(3), 247-259. https://doi.org/10.1023/A:1023910315561

van Rijsoort, S., Emmelkamp, P., \& Vervaeke, G. (1999). The Penn State Worry Questionnaire and the Worry Domains Questionnaire: Structure, reliability and validity. Clinical Psychology \& Psychotherapy, 6(4), 297-307. https://doi.org/10.1002/(SICI)10990879(199910)6:4<297::AID-CPP206>3.0.CO;2-E

Vanderhasselt, M.-A., Brose, A., Koster, E. H. W., \& Raedt, R. de (2016). Co-variation between stressful events and rumination predicts depressive symptoms: An eighteen months prospective design in undergraduates. Behaviour Research and Therapy, 87, 128133. https://doi.org/10.1016/j.brat.2016.09.003

Verstraeten, K., Bijttebier, P., Vasey, M. W., \& Raes, F. (2011). Specificity of worry and rumination in the development of anxiety and depressive symptoms in children. British Journal of Clinical Psychology, 50(4), 364-378.

https://doi.org/10.1348/014466510X532715 
Wagner, C. A., Alloy, L. B., \& Abramson, L. Y. (2015). Trait rumination, depression, and executive functions in early adolescence. Journal of Youth and Adolescence, 44(1), 18-36. https://doi.org/10.1007/s10964-014-0133-8

Watkins, E. (2004). Appraisals and strategies associated with rumination and worry. Personality and Individual Differences, 37(4), 679-694. https://doi.org/10.1016/j.paid.2003.10.002

Watkins, E. (2008). Constructive and unconstructive repetitive thought. Psychological Bulletin, 134(2), 163-206. https://doi.org/10.1037/0033-2909.134.2.163

Watkins, E., Moulds, M., \& Mackintosh, B. (2005). Comparisons between rumination and worry in a non-clinical population. Behaviour Research and Therapy, 43(12), 1577-1585. https://doi.org/10.1016/j.brat.2004.11.008

Watkins, E., Teasdale, J. D., \& Williams, R. M. (2000). Decentring and distraction reduce overgeneral autobiographical memory in depression. Psychological Medicine, 30(4), 911920. https://doi.org/10.1017/S0033291799002263

Xu, R. (2003). Measuring explained variation in linear mixed effects models. Statistics in Medicine, 22(22), 3527-3541. https://doi.org/10.1002/sim.1572

Yang, Y., Cao, S., Shields, G. S., Teng, Z., \& Liu, Y. (2017). The relationships between rumination and core executive functions: A meta-analysis. Depression and Anxiety, 34(1), 37-50. https://doi.org/10.1002/da.22539

Yuan, K.-H., \& Bentler, P. M. (2000). 5. Three likelihood-based methods for mean and covariance structure analysis with nonnormal missing data. Sociological Methodology, 30(1), 165-200. https://doi.org/10.1111/0081-1750.00078 
Yung, Y.-F., Thissen, D., \& McLeod, L. D. (1999). On the relationship between the higherorder factor model and the hierarchical factor model. Psychometrika, 64(2), 113-128. https://doi.org/10.1007/BF02294531

Zetsche, U., Bürkner, P.-C., \& Schulze, L. (2018). Shedding light on the association between repetitive negative thinking and deficits in cognitive control - A meta-analysis. Clinical Psychology Review, 63, 56-65. https://doi.org/10.1016/j.cpr.2018.06.001

Ziegert, D. I., \& Kistner, J. A. (2002). Response Styles Theory: Downward extension to children. Journal of Clinical Child and Adolescent Psychology, 31(3), 325-334. https://doi.org/10.1207/153744202760082586

Zoccola, P. M., Dickerson, S. S., \& Yim, I. S. (2011). Trait and state perseverative cognition and the cortisol awakening response. Psychoneuroendocrinology, 36(4), 592-595. https://doi.org/10.1016/j.psyneuen.2010.10.004 
Table 1

Descriptive Statistics of the Study Variables

\begin{tabular}{|c|c|c|c|c|c|c|c|c|c|}
\hline \multirow{2}{*}{ Variable } & \multirow{2}{*}{$M(S D)$} & \multirow[t]{2}{*}{$\mathrm{ICC}$} & \multirow{2}{*}{ Range } & \multicolumn{6}{|c|}{ Correlations } \\
\hline & & & & 2 & 3 & 4 & 5 & 6 & 7 \\
\hline \multicolumn{10}{|l|}{ Study 1} \\
\hline 1. Age & $9.88(0.60)$ & - & $8-11$ & -.17 & -.04 & .00 & .25 & -.02 & \\
\hline 2. Gender ${ }^{\mathrm{a}}$ & $.41(0.49)$ & - & 0-1 & - & -.08 & .06 & .09 & .05 & \\
\hline 3. Negative affect & $1.54(0.58)$ & .50 & $1.00-5.00$ & & - & -.29 & -.37 & .47 & \\
\hline 4. Positive affect & $4.04(0.77)$ & .57 & $1.00-5.00$ & & -.17 & - & .28 & -.10 & \\
\hline 5. Working memory & $.61(0.21)$ & .62 & $0.00-1.00$ & & -.18 & .13 & - & -.19 & \\
\hline 6. Rumination & $1.84(0.70)$ & .43 & $1.00-5.00$ & & .18 & -.01 & .03 & - & \\
\hline \multicolumn{10}{|l|}{ Study 2} \\
\hline 1. Age & $8.98(0.77)$ & - & $8-10$ & -.00 & -.09 & -.17 & -.09 & -.09 & -.02 \\
\hline 2. Gender ${ }^{\mathrm{a}}$ & $.44(0.50)$ & - & $0-1$ & - & -.07 & .08 & .04 & -.04 & .04 \\
\hline 3. Negative affect & $1.42(0.47)$ & .52 & $1.00-5.00$ & & & -.08 & -.38 & .66 & .77 \\
\hline 4. Positive affect & $3.67(0.72)$ & .58 & $1.00-5.00$ & & -.16 & - & .08 & .09 & -.01 \\
\hline 5. Working memory & $.73(0.14)$ & .49 & $0.00-1.00$ & & -.04 & .12 & - & -.33 & -.33 \\
\hline 6. Rumination & $1.74(0.86)$ & .57 & $1.00-5.00$ & & .28 & -.01 & .04 & - & .69 \\
\hline 7. Worry & $1.38(0.54)$ & .46 & $1.00-5.00$ & & .58 & -.05 & .00 & .36 & - \\
\hline
\end{tabular}

Note. Time-varying variables were aggregated on the day level. Correlations on the between-person level are depicted above the diagonal; correlations on the within-person level are shown below the diagonal. Statistically significant correlations are printed in bold. ICC $=$ Intraclass Correlation Coefficient; Study 1: $N=110$; Study 2: $N=84$.

${ }^{\mathrm{a}} 0=$ male; 1 = female. 
Table 2

Results of Multilevel Models Predicting Negative Affect, Positive Affect and Working Memory Performance From Rumination

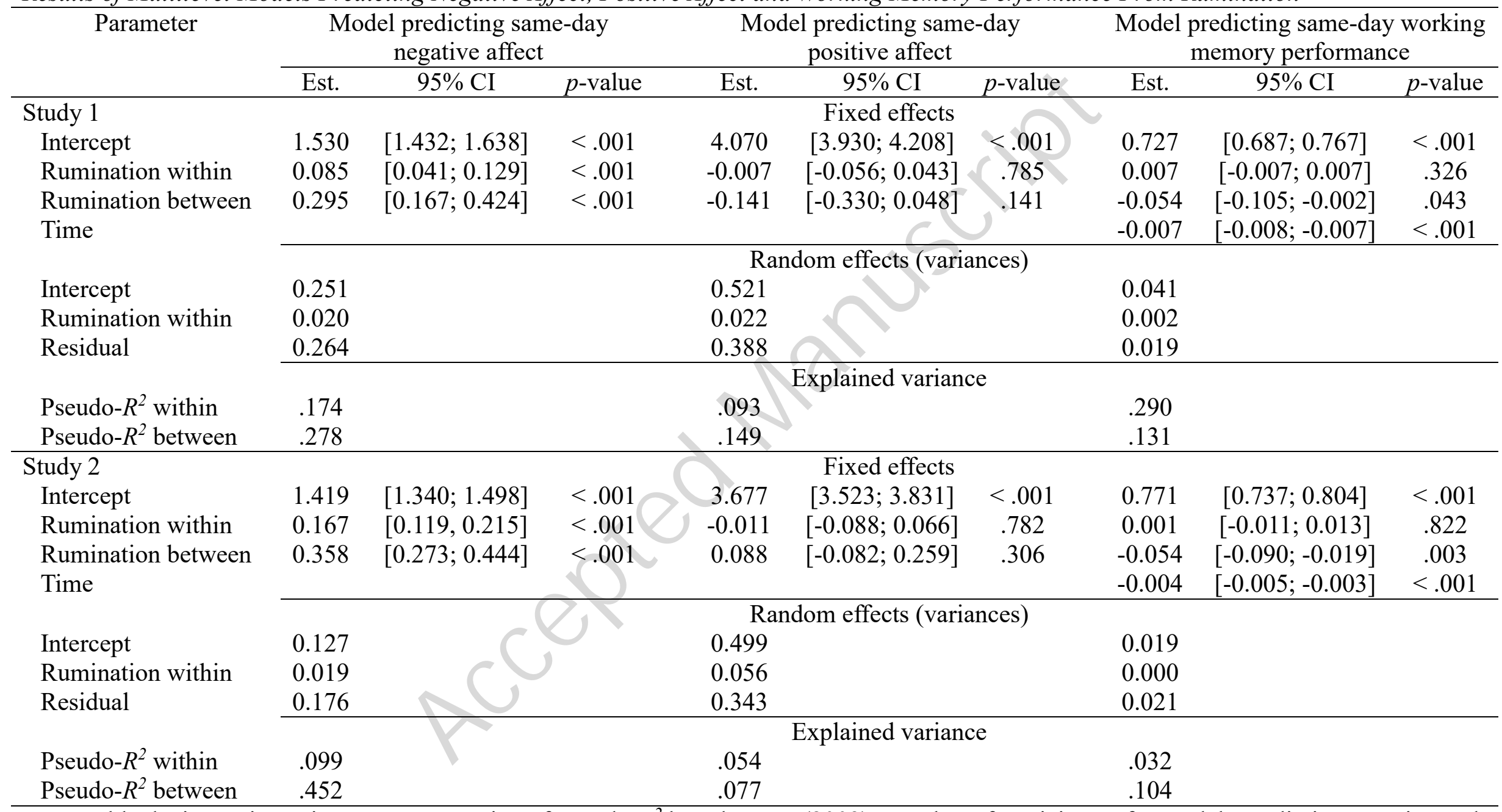

Note. Table depicts point estimates. Computation of Pseudo- $R^{2}$ based on Xu (2003). Number of participants for models predicting negative and positive affect in Study $1=110$; number of participants for the model predicting working memory performance in Study $1=109$; number of 
participants for all models in Study $2=84$; number of observations in Study 1 for (a) the model predicting negative affect $=1967$, (b) the model predicting positive affect $=1969$, (c) the model predicting working memory performance $=1873$; number of observations in Study 2 for (a) the model predicting negative affect $=1558$, (b) the model predicting positive affect $=1558$, (c) the model predicting working memory performance $=1284$; Est. $=$ Estimate; $\mathrm{CI}=$ Confidence Interval. 
Table 3

Results of Multilevel Models Predicting Negative Affect, Positive Affect and Working Memory Performance From Worry

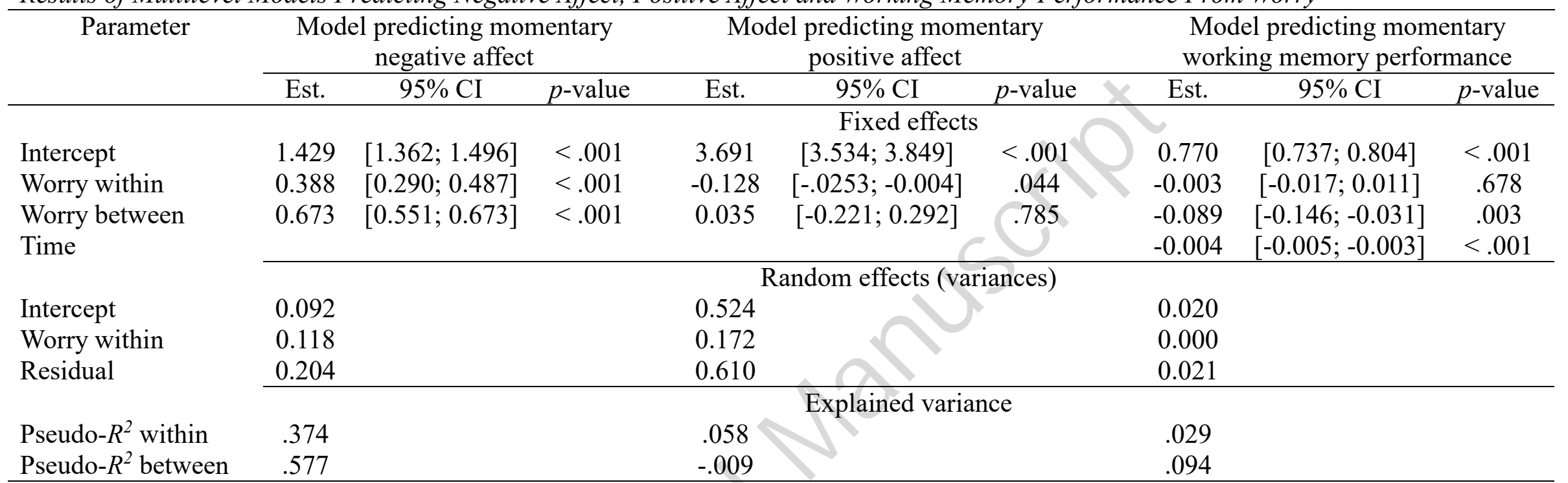

Note. Table depicts point estimates. Computation of Pseudo- $R^{2}$ based on Xu (2003). Number of participants for all models = 84; number of observations for (a) the model predicting negative affect $=2959$, (b) the model predicting positive affect $=2960$, (c) the model predicting working memory performance $=1332$; Est. = Estimate; $\mathrm{CI}=$ Confidence Interval. 


\section{Figure 1}

Factor Models for the two Short Scales on the Within- and Between-Person Level

a)

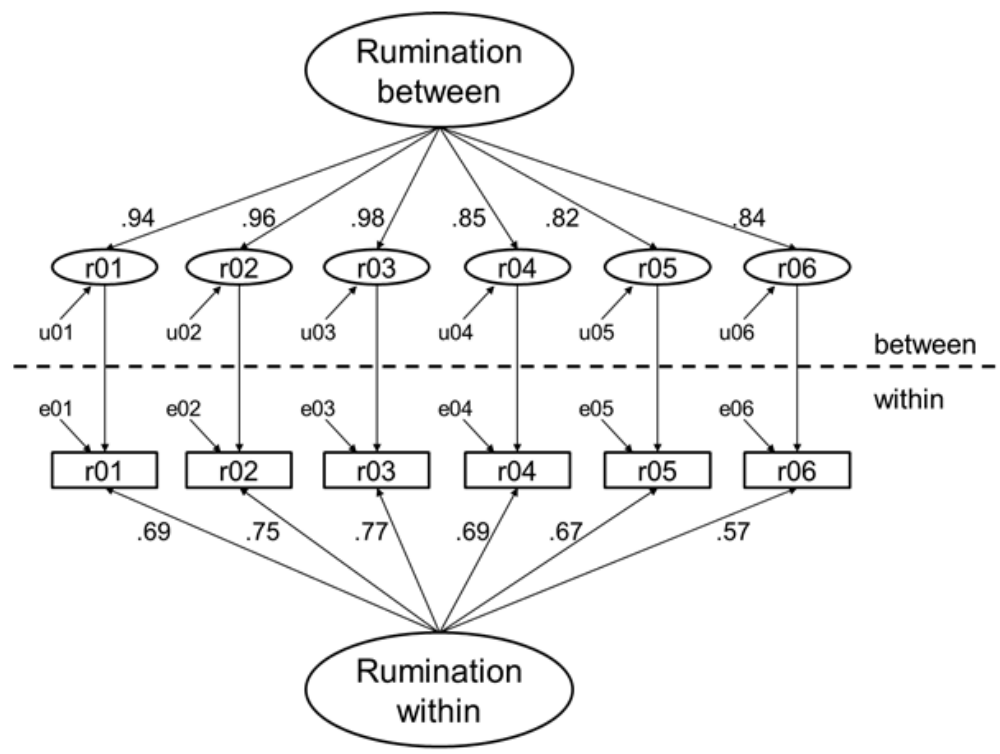

b)

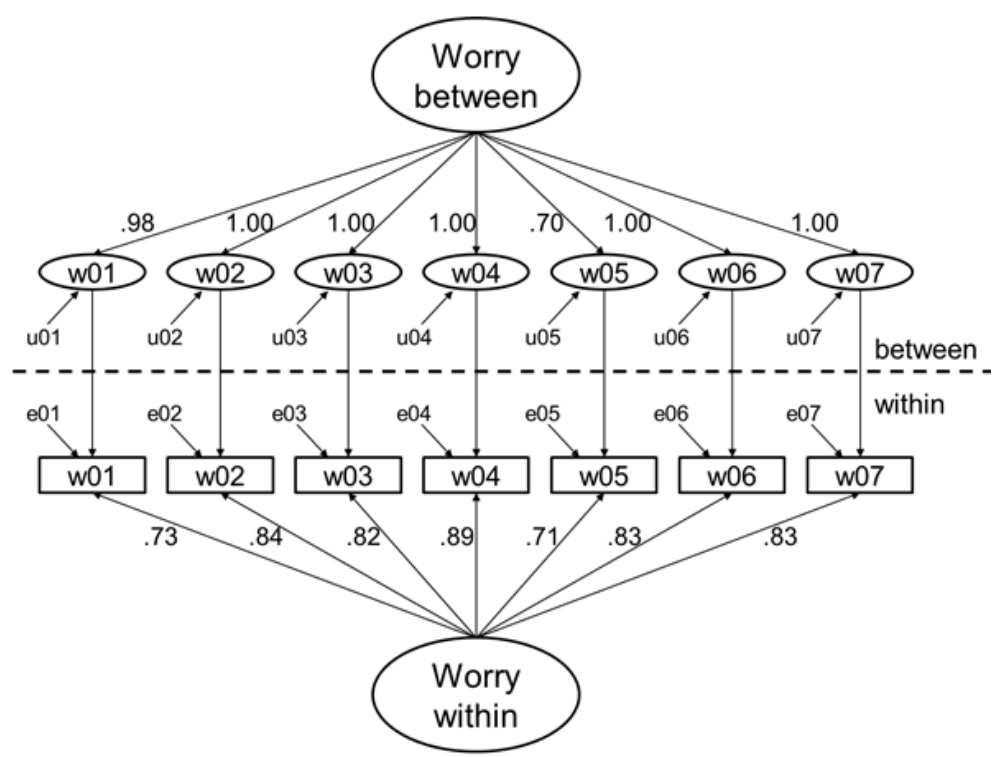

Note. Figure 1a depicts the model for the ARS in Study 1. Figure 1b shows the model for the AWS in Study 2. Coefficients represent standardized factor loadings. 